\title{
Self-calibrating Tomography for Angular Schmidt Modes in Spontaneous Parametric Down-Conversion
}

\author{
S.S.Straupe圈 and D.P.Ivanov ${ }^{1}$, A.A.Kalinkin ${ }^{1}$, I.B.Bobrov ${ }^{1}$, S.P.Kulik ${ }^{1}$ and D.Mogilevtsev ${ }^{2,3}$ \\ ${ }^{1}$ Faculty of Physics, M.V.Lomonosov Moscow State University, 199001, Moscow, Russia \\ ${ }^{2}$ Centro de Ciências Naturais e Humanas, Universidade Federal do ABC, Santo André, SP, 09210-170 Brazil; \\ ${ }^{3}$ Institute of Physics, Belarus National Academy of Sciences, F.Skarina Ave. 68, Minsk 220072 Belarus
}

(Dated: November 10, 2018)

\begin{abstract}
We report an experimental self-calibrating tomography scheme for entanglement characterization in high-dimensional quantum systems using Schmidt decomposition techniques. The selftomography technique based on maximal likelihood estimation was developed for characterizing non-ideal measurements in Schmidt basis allowing us to infer both Schmidt eigenvalues and detecting efficiencies.
\end{abstract}

PACS numbers: 03.65.Wj, 42.50.Lc, 03.67.Bg, 03.67.Mn, 42.65.Lm

\section{INTRODUCTION}

Building an experimental set-up for performing sophisticated measurements (for example, such as ones required for performing quantum tomography), one generally needs calibrating it. For the signals on the singleparticle level the task is quite challenging, especially if one cannot easily use a pre-calibrated etalon detectors and/or signal sources for the purpose. More than 30 years ago D. N. Klyshko had outlined an efficient way to solve this problem using the fact that quantum features of the signal (such as, for example, character of the photon number distribution) can be used as a precise measurement tool for a calibration. In particular, D. N. Klyshko had suggested using the fact of photon pairs creation in the process of down-conversion for performing an "absolute calibration" of the detecting scheme [1]. A detection of one photon of the pair in one arm of the "absolute calibration" scheme means that there is the second photon of the pair going through the other arm. Thus, the ratio of registered counts in both arms gives an experimentalist efficiency of the detecting set-up installed in the second arm of the scheme without any pre-calibrated detector/source. This idea was actively developed and implemented (see, for example, Refs. [2 [5]). Recently, this idea had also given rise to more general concept of "self-calibration" as simultaneous inference of parameters of both the measurement scheme and the signal [68]. Very recently the first experimental realization of the self-calibration scheme was presented using polarizationencoded one- and two-photon states 9]. There, the unknown rotation angle of the measurement basis was recovered together with the density matrix of the signal.

Here we present the first example of experimental selfcalibrating tomography, when a set of parameters describing efficiency of the detecting scheme is actually inferred together with the parameters of the spatial state of

*Electronic address: straups@yandex.ru entangled photon pairs generated in the process of spontaneous parametric down-conversion (SPDC).

Spatial entanglement in SPDC was a subject of intense research during the last decade. Besides fundamental issues, spatial states of biphoton pairs offer a platform for high-dimensional quantum states engineering motivating this interest. One can distinguish two complementary approaches to spatial qudit engineering with biphotons: one using "pixel entanglement" and similar schemes 10 13], and another one based on using high-order coherent (usually Laguerre-Gaussian) modes [14 25]. In both approaches achievable dimensionality and collection efficiency are figures of merit. Dimensionality of effective Hilbert space is limited by degree of spatial entanglement. In pixel entanglement schemes, for example, the pixel size should be made smaller than the coherence radius of the pump in the far zone, and since the pump is always divergent, even a plain wave, selected by point-like aperture would be correlated to a whole set of plain-wave modes in the conjugate beam. The same holds in general for other possible choices of modes.

It is remarkable that there is a "preferred" basis among the multitude of possible coherent spatial modes, which consists only of pairwise correlated modes. It is a set of Schmidt modes. Since it was used for the first time by Law and Eberly 26], it has become a common tool for entanglement analysis of infinite dimensional systems in general, and of spatial states of photons in particular. A direct experimental attempt to address spatial entanglement of SPDC biphotons in Schmidt basis was made in the recent work of authors 27]. The technique of projective measurements used in that work suffers from poor quality of spatial mode transformations, resulting in non-ideal measurement scheme. Here we use selfcalibration to account for this non-ideality. We present a self-consistent analysis of the data collected by measuring approximate Schmidt modes via the set-up similar to Ref.[27], and demonstrate that self-calibrating tomography is a feasible and practical way to update both the information about the measurement scheme and Schmidt eigenvalues starting from very general assumptions about them. 
The paper is organized as follows: in Section II we briefly describe the main features of SPDC angular spectrum with emphasis on spatial entanglement and Schmidt decomposition, Section III describes the general concept of the self-calibrating tomography scheme and its particular realization for inferring Schmidt eigenvalues. Section IV gives the detailed description of our experiments with spatial Schmidt modes. Section $\mathrm{V}$ describes the practical implementation of the selfcalibration scheme.

\section{ANALYZING SPATIAL ENTANGLEMENT OF SPDC BIPHOTONS WITH SCHMIDT DECOMPOSITION}

Biphotons generated in the SPDC process have continuous frequency and angular spectrum. Let us consider its structure in some details. SPDC can be phenomenologically described using the following effective interaction Hamiltonian [28]:

$$
\left.H=\int_{V} d^{3} \vec{r} \chi^{(2)}(\vec{r}) E_{p}^{(-)}(\vec{r}) E^{(+)}(\vec{r})\right) E^{(+)}(\vec{r})+\text { H.c. }
$$

Here $E_{p}$ is the classical amplitude of the pump field and $E$ is the scattered field operator. Considering pump to be monochromatic, the first order of perturbation theory gives the following expression for the state of the scattered field:

$$
\begin{aligned}
& |\Psi\rangle=|v a c\rangle+\int d \overrightarrow{k_{1}} d \overrightarrow{k_{2}} \Psi\left(\overrightarrow{k_{1}}, \overrightarrow{k_{2}}\right)|1\rangle_{k_{1}}|1\rangle_{k_{2}}, \\
& \Psi\left(\overrightarrow{k_{1}}, \overrightarrow{k_{2}}\right)=\int_{V} d^{3} \overrightarrow{r^{\prime}}{ }^{(2)}(\vec{r}) E_{p}^{(-)}(\vec{r}) \exp [i \vec{\Delta} \vec{r}],
\end{aligned}
$$

where $\vec{\Delta}=\overrightarrow{k_{1}}+\overrightarrow{k_{2}}-\overrightarrow{k_{p}}, \omega_{1}+\omega_{2}=\omega_{p}$. In the case of collinear phase-matching and under wide crystal approximation one can obtain the following biphoton field amplitude 29 31]:

$$
\Psi\left(\overrightarrow{k_{1}}, \overrightarrow{k_{2}}\right)=\mathcal{E}_{p}\left(\overrightarrow{k_{1 \perp}}+\overrightarrow{k_{2 \perp}}\right) \mathcal{F}\left(\overrightarrow{k_{1 \perp}}-\overrightarrow{k_{2 \perp}}\right),
$$

where $\mathcal{E}_{p}\left(\overrightarrow{k_{1 \perp}}+\overrightarrow{k_{2 \perp}}\right)$ stands for angular spectrum of the pump, and $\mathcal{F}\left(\overrightarrow{k_{1}}-\overrightarrow{k_{2}}\right)$ is a geometrical factor determined by phase-matching conditions.

Authors of [30, 31] give the following expression for $\mathcal{F}$ :

$$
\Psi\left(\overrightarrow{k_{1}}, \overrightarrow{k_{2}}\right)=\mathcal{N} \mathcal{E}_{p}\left(\overrightarrow{k_{1 \perp}}+\overrightarrow{k_{2 \perp}}\right) \operatorname{sinc}\left[\frac{L\left(\overrightarrow{k_{1 \perp}}-\overrightarrow{k_{2 \perp}}\right)^{2}}{4 k_{p}}\right] \text {, }
$$

with $L$ being the crystal length and $\mathcal{N}$ - a normalization constant. Although this expression is strictly valid only in the case of small pump divergence [13, 32], it appropriately describes the SPDC spectrum in our experiments.

The most developed approach to quantitative analysis of spatial (and frequency) entanglement of SPDC biphoton states is based on using coherent modes decomposition. Biphoton spatial state space is "discretized" by switching from continuous distributions in plane-wave basis of the previous section to discrete distributions in a chosen basis of spatial mode functions $\xi_{i}\left(\vec{k}_{1 \perp, 2 \perp}\right)$. For an arbitrary choice of mode functions in the decomposition of spatial state for each of the photons, the biphoton amplitude takes the following form:

$$
\Psi\left(\vec{k}_{1 \perp}, \vec{k}_{2 \perp}\right)=\sum_{i, j=0}^{\infty} C_{i j} \xi_{i}\left(\vec{k}_{1 \perp}\right) \xi_{j}\left(\vec{k}_{2 \perp}\right) .
$$

It turns out, that by appropriate choice of the basis mode functions one can transform the expression (5) to a single-sum form

$$
\Psi\left(\vec{k}_{1 \perp}, \vec{k}_{2 \perp}\right)=\sum_{i=0}^{\infty} \sqrt{\lambda_{i}} \psi_{i}\left(\vec{k}_{1 \perp}\right) \psi_{i}\left(\vec{k}_{2 \perp}\right)
$$

which is called Schmidt decomposition. In this case the basis functions $\psi_{i}\left(\vec{k}_{1, \perp}\right)$ should be eigenfunctions of single-photon density matrix $\rho_{1,2}\left(\vec{k}_{1 \perp}, \vec{k}_{1 \perp}\right)$, and $\lambda_{i}$ are the corresponding eigenvalues. It means that the appropriate mode functions may be found by solving the following integral equation:

$$
\int \rho_{1,2}\left(\vec{k}_{\perp}, \vec{k}_{\perp}^{\prime}\right) \psi_{i}\left(\vec{k}_{\perp}^{\prime}\right) \mathrm{d} \vec{k}_{\perp}^{\prime}=\lambda_{i} \psi_{i}\left(\vec{k}_{\perp}\right) .
$$

"Average number of Schmidt modes" determined by Schmidt number:

$$
K=\frac{1}{\sum_{i=0}^{\infty} \lambda_{i}^{2}}
$$

is widely used as an operational measure of entanglement 26].

Degree of spatial entanglement of SPDC biphotons described by wavefunction (4) was analyzed by Law and Eberly in [26]. The pump was assumed to be gaus$\operatorname{sian} \mathcal{E}_{p}=\exp \left[-\frac{\left|\overrightarrow{k_{1}}+k_{2 \perp}\right|}{\sigma^{2}}\right]$. Authors derived an analytical expression for Schmidt number by approximating $\mathcal{F}\left(\overrightarrow{k_{1 \perp}}-\overrightarrow{k_{2 \perp}}\right)$ function of (4) by a gaussian function:

$$
K_{g}=\frac{1}{4}\left(b \sigma+\frac{1}{b \sigma}\right)^{2},
$$

where $b=\sqrt{\frac{L}{4 k_{p}}}$ is the waist of the gaussian function modeling $\mathcal{F}\left(\overrightarrow{k_{1 \perp}}-\overrightarrow{k_{2 \perp}}\right)$. In the following we will call this procedure "a double gaussian approximation". The value of $K_{g}$ is determined by a single parameter $b \sigma$ and may reach very high values for $b \sigma \gg 1$ and $b \sigma \ll 1$. Numerical calculations performed for

$$
\mathcal{F}\left(\overrightarrow{k_{1 \perp}}-\overrightarrow{k_{2 \perp}}\right)=\operatorname{sinc}\left[b\left(\overrightarrow{k_{1 \perp}}-\overrightarrow{k_{2 \perp}}\right)^{2}\right]
$$

showed that real value of $K$ is larger than $K_{g}$ for all values of $b \sigma$. 
The choice of mode functions set in (5) is quite arbitrary and may be determined by convenience in analyzing a particular physical situation. If the situation corresponds to a beam propagating in free space, it is natural to chose the solutions of paraxial wave equation, i.e. Hermite-Gaussian or Laguerre-Gaussian modes, as a set of mode functions. Moreover it turns out, that these functions form the Schmidt decomposition for SPDC with the gaussian pump of moderate divergence.

Let us choose the set of Hermite-Gaussian modes as a basis for decomposition:

$$
\begin{aligned}
& \mathrm{HG}_{n m}\left(k_{x}, k_{y}\right) \propto \\
& \mathrm{H}_{n}\left(\frac{k_{x}^{2}}{\left(\Delta k_{x}\right)^{2}}\right) \mathrm{H}_{m}\left(\frac{k_{y}^{2}}{\left(\Delta k_{y}\right)^{2}}\right) \exp \left(-\frac{k_{x}^{2}+k_{y}^{2}}{2\left(\Delta k_{\perp}\right)^{2}}\right),
\end{aligned}
$$

where $\mathrm{H}_{n}(x)$ are Hermite polynomials, and $\left\{k_{x}, k_{y}\right\}$ are transverse wave-vector components. One can get rid of two indexes in decomposition (11) and transform it to a form of Schmidt decomposition using double-gaussian approximation [26, 34]. For small $\sigma$ we can make a substitution $\operatorname{sinc}\left(\frac{x^{2}}{\sigma^{2}}\right) \rightarrow \exp \left(-\gamma \frac{x^{2}}{\sigma^{2}}\right)$, where $\gamma$ is a coefficient chosen to make both functions "close" to each other. A good approximation is provided by choosing a value of $\gamma=0.86$. 42] The biphoton wavefunction now takes the following form:

$\Psi\left(\overrightarrow{k_{1}}, \overrightarrow{k_{2}}\right) \propto \exp \left(-\frac{\left(\overrightarrow{k_{1 \perp}}+\overrightarrow{k_{2 \perp}}\right)^{2}}{2 a^{2}}\right) \exp \left(-\frac{\left(\overrightarrow{k_{1 \perp}}-\overrightarrow{k_{2 \perp}}\right)^{2}}{2 b^{2}}\right)$,

where $a$ determines the angular bandwidth of the pump, and $b=\sqrt{4 k_{p} / \gamma L}$ - the phase-matching bandwidth. Since the wavefunction is a product of functions depending only on $k_{1,2 x}$ and only on $k_{1,2 y}$, it is sufficient to consider the problem in one dimension:

$$
\begin{aligned}
& \Psi\left(k_{1 x}, k_{2 x}\right)= \\
& \sqrt{\frac{2}{\pi a b}} \exp \left(-\frac{\left(k_{1 x}+k_{2 x}\right)^{2}}{2 a^{2}}\right) \exp \left(-\frac{\left(k_{1 x}-k_{2 x}\right)^{2}}{2 b^{2}}\right) .
\end{aligned}
$$

One can show, that solutions of (7) for such a wavefunction have the form [34]:

$$
\psi_{n}\left(k_{1 x, 2 x}\right)=\left(\frac{2}{a b}\right)^{1 / 4} \phi_{n}\left(\sqrt{\frac{2}{a b}} k_{1 x, 2 x}\right),
$$

where $\phi_{n}(x)=\left(2^{n} n ! \sqrt{\pi}\right)^{-1 / 2} e^{-x^{2} / 2} \mathrm{H}_{n}(x)$. For corresponding eigenvalues and Schmidt number we obtain:

$$
\lambda_{n}=4 a b \frac{(a-b)^{2 n}}{(a+b)^{2(n+1)}}, \quad K_{x}=\frac{a^{2}+b^{2}}{2 a b} .
$$

So we have the following form of Schmidt decomposition for SPDC biphoton state under the double-gaussian approximation:

$$
\begin{aligned}
& \Psi\left(\overrightarrow{k_{1}}, \overrightarrow{k_{2}}\right)= \\
& \sum_{m n} \sqrt{\lambda_{n} \lambda_{m}} \psi_{n}\left(k_{1 x}\right) \psi_{m}\left(k_{1 y}\right) \times \psi_{n}\left(k_{2 x}\right) \psi_{m}\left(k_{2 y}\right) .
\end{aligned}
$$

Degree of entanglement for this two-dimensional wavepacket is given by Schmidt number:

$$
K=K_{x} \times K_{y}=\left(a^{2}+b^{2}\right)^{2} / 4 a^{2} b^{2} .
$$

Let us note, that (16) is not the only possible form of Schmidt decomposition. It may as well be described in terms of Laguerre-Gaussian modes as in 26, 35]. The value of Schmidt number is, of course, basis independent, as was explicitly shown in a recent preprint by Miatto et al. [36]. In fact, the difference between these two representations corresponds to the choice of polar or cartesian coordinates on the plane of transverse momentum components.

\section{SELF-CALIBRATING TOMOGRAPHY SCHEME FOR INFERRING SCHMIDT EIGENVALUES}

Here we describe briefly the concept of self-calibration as simultaneous updating of information about the state and the measurement device as it was formulated in a recent work [8]. Also, we discuss the application of the selfcalibration scheme for tomography of angular Schmidt modes in SPDC.

Generally, the possibility of self-calibration follows naturally from the Born rule

$$
p(\rho, X)=\operatorname{Tr}\{\hat{\Pi}(X) \rho\},
$$

which gives one a probability of getting the particular measurement result, $p(\rho, X)$, being linear on coefficients of the representation of the signal density matrix, $\rho$, and the elements of the Positive Operator Valued Measure (POVM), $\hat{\Pi}(X)$, in an arbitrary basis. Obviously, a-priori knowledge of some parts of the signal density matrix can be traded for getting knowledge of some parts of the POVM. And even if one aims for inferring such parameters of the POVM (say, $X$ in Eq.(18)) that the probability, $p(\rho, X)$, depends nonlinearly on, both $X$ and the signal density matrix, it can be estimated, provided that a measure of estimation success is strictly convex with respect to all the parameters/coefficients to be found. For this measure one can take, for example, the KullbackLeibler divergence between the expected inferred set of probabilities, $\vec{p}=\left\{p_{i j}(\rho, X)\right\}$, and the actually measured frequencies, $\vec{f}=\left\{f_{i j}\right\}[8]$,

$$
\begin{array}{r}
D(\vec{p}, \vec{f}) \propto \ln (L)=\sum_{i, j} f_{i j} \ln \left\{\frac{p_{i j}(\rho, X)}{P}\right\}, \\
P=\sum_{i j} p_{i j}(\rho, X) .
\end{array}
$$

Minimizing this divergence is equivalent to performing maximum-likelihood (ML) estimation [38], i.e. maximizing the likelihood $L$ of the model.

Building the self-calibration statistical estimation procedure for our case is greatly simplified, firstly, by the 
available a-priori knowledge about the signal state and, secondly, by the character of measurements. Actually, one needs estimating diagonal elements of the density matrix (6) containing terms corresponding to no more than single photons. This allows one implementing highly efficient iterative expectation-maximization algorithm for performing ML estimation of the diagonal elements of the density matrix, preserving positivity on each step of the iteration procedure 39] (for practical implementation for the diagonal elements estimation see, for the example, Refs. [6, 40]). Also, as it will be seen below, the measurement is done by performing rather accurate (albeit still significantly non-ideal) projection on the chosen Schmidt mode approximated as the Gaussian function (see the previous Section). Local measurements in one of directions are sufficient for the inference.

So, the reduced local signal density matrix describing one photon of the pair is

$$
\rho\left(\vec{k}, \overrightarrow{k^{\prime}}\right)=\sum_{n, m} \lambda_{m n} \psi_{n m}(\vec{k}) \psi_{n m}\left(\overrightarrow{k^{\prime}}\right)
$$

where coefficients $\lambda_{n m}=\lambda_{m} \lambda_{n}$ are Schmidt numbers corresponding to the mode $\psi_{n m}$. The POVM elements for the local projective measurement of the mode $\psi_{n m}$ can be written as

$$
\Pi_{n m}\left(\vec{k}, \overrightarrow{k^{\prime}}\right)=\sum_{i, j} \mu_{i j}^{(n m)} \psi_{i j}(\vec{k}) \psi_{i j}\left(\overrightarrow{k^{\prime}}\right),
$$

where parameters $\mu_{i j}^{(n m)}$ describe losses and efficiency of the projection. Knowledge about these parameters is to be updated via the self-calibration procedure. The expectation-maximization iterative procedure to the case can be written as:

$$
\lambda_{n m}^{(k+1)}=\lambda_{n m}^{(k)} \sum_{i, j} \frac{f_{i j}}{p_{i j}^{(k)}} \frac{\mu_{i j}^{(n m)}}{\mu_{i j}^{s u m}},
$$

where

$$
\mu_{i j}^{s u m}=\sum_{n, m} \mu_{i j}^{(n m)}
$$

and probabilities $p_{i j}^{(k)}$ are estimated via the Born rule (18) on the $k$ th iteration of the procedure. For the initial approximation, $\lambda_{m n}^{(0)}$, one can choose, for example, the set of equal numbers (really, the choice of $\lambda_{m n}^{(0)}$ is not really important provided for that they are non-zero [40]). An important feature of the procedure (22) specific to our case is that the result of estimation should be factorable, $\lambda_{n m}^{(k)}=\lambda_{m}^{(k)} \lambda_{n}^{(k)}$. Practically, it can be done by replacing the result of each iteration by the closest factorable matrix. As we shall see below, the procedure (22) provides for fast and efficient estimation of Schmidt eigenvalues.

The self-calibration procedure can be imagined as follows: one assumes some values of parameters $\mu_{i j}^{(n m)}$, estimates $\lambda_{m n}$ and probabilities $p_{i j}$ and calculates the value of the Kullback-Leibler divergence (19). Then one repeats the whole procedure for another set of $\mu_{i j}^{(n m)}$. If the Kullback-Leibler divergence is convex in the chosen region of parameters $\mu_{i j}^{(n m)}$, one chooses the values of $\mu_{i j}^{(n m)}$ corresponding to the minimum divergence as ones allowing the closest fitting of the experimental results.

\section{EXPERIMENTAL REALIZATION OF MEASUREMENTS IN SCHMIDT BASIS}

Here we describe locals measurement performed by projection to the approximated Gaussian Schmidt modes.

We used a $2 \mathrm{~mm}$ BBO crystal pumped by a CW HeCd laser with $\lambda_{p}=325 \mathrm{~nm}$ wavelength. The crystal was cut for collinear frequency-degenerate Type-I phasematching. Angular bandwidth of phase-matching in such crystal (neglecting the pump divergence) $-b$ parameter in (12) is $\frac{\lambda}{2 \pi} \times b=0.033$, where $\lambda=2 \lambda_{p}=650 \mathrm{~nm}$ is the wavelength of down-converted photons. It was convenient for our purposes to select the value of pump divergence corresponding to a moderate Schmidt number. We focused the pump inside the crystal with a $150 \mathrm{~mm}$ quartz lens and measured the divergence - $a$ parameter in (12) to be $\frac{\lambda}{2 \pi} \times a=(5.8 \pm 0.1) \times 10^{-3}$ [43]. To ensure applicability of double-gaussian approximation we calculated eignevalues and eigenfunctions for the reduced single-photon density matrix, corresponding to the precise SPDC wavefunction (4) numerically. The calculation was performed as follows: Hermite-Gaussian modes corresponding to the approximate function (13) were chosen as a basis, we have restricted ourselves to 10 lower order modes (giving the Schmidt number with 3 decimal digits precision) and calculated the matrix elements of the precise density matrix in this basis. Diagonalizing the calculated matrix, we obtained eigenvalues and eigenfunctions. The results are in reasonable correspondence with a simplified double-gaussian model, at least, we should expect that phase holograms for Schmidt modes should be close to those of Hermite-Gaussian modes of appropriate divergence. We should note that measured waist size of the pump beam in the focal plane of the lens was $w_{p}=(25 \pm 1) \mu \mathrm{m}$, corresponding to $M^{2}=1.4$. That means, the pump beam is aberrated and is not really gaussian, and that may cause some deviations from Hermite-Gaussian shape of Schmidt modes as well.

We have used an LCoS SLM with VAN matrix produced by Cambridge Correlators. The matrix has $1027 \times$ 768 pixels of $10 \mu \mathrm{m}$ size each. It is an 8 bit device, capable of introducing a phase shift of up to $0.8 \pi$. Since larger phase shifts are required for our holograms we used a double reflection scheme. We used two polymer film polarizers in front and after the SLM to reduce the unwanted polarization rotations by an additional dielectric mirror necessary in such scheme (see insets in Fig. 15).

To estimate the quality of quality of mode transfor- 


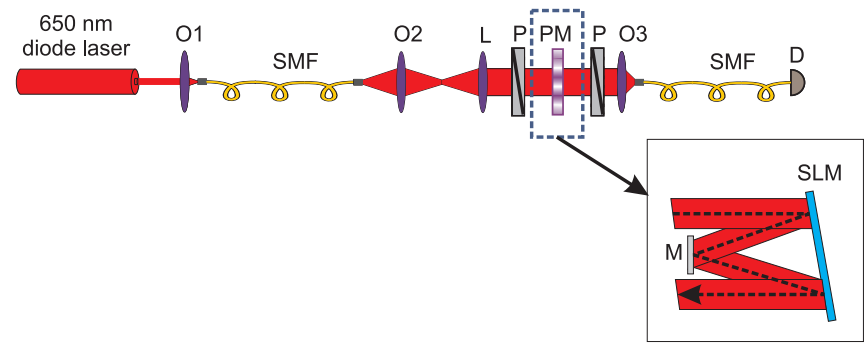

Figure 1: Experimental setup for transforming an attenuated laser beam to higher Hermite-Gaussian modes. SMF - single mode fiber; O1,2,3 - microscope objectives; L - 145 mm lens; $\mathrm{P}$ - polarizers; D - single photon counter. A phase modulator is shown as a transmitting mask, while in reality it is a combination of SLM and a dielectric mirror M (see text for details).

mation with this device we used the setup sketched in Fig. 1. We used an attenuated $650 \mathrm{~nm}$ diode laser as a source. The beam was mode filtered with single mode fiber and focused with a $20 \times$ microscope objective to obtain the divergence similar to that of an $H G_{00}$ Schmidt mode, and the waist at the position of the crystal. So we obtained a single mode gaussian beam modeling the zero order Schmidt mode of SPDC beam. The beam was collimated with $145 \mathrm{~mm}$ lens and after reflection from SLM was focused with a $8 \times$ microscope objective to a single mode fiber followed by a single photon counter (Perkin Elmer). The focused beam waist exactly coincided with the mode size of the fiber $(4 \mu \mathrm{m})$. We should stress, that we paid special attention to mode matching and optics were chosen in such a way that detection mode exactly coincides with calculated $H G_{00}$ Schmidt mode. Parameters of phase holograms were adjusted to minimize the detector counting rate, i.e. to ensure orthogonality of transformed modes to a fundamental gaussian one. We have actually adjusted three parameters: the position of phase step for $H G_{10(01)}$ modes, which is determined by the beam position at the SLM (in horizontal and vertical directions, respectively), and the distance between phase steps for $H G_{20}$ modes determined by beam size at the SLM plane. These parameters define the shape of holograms for other modes in a unique way. If we define "visibility" for mode transformations as the ratio of counting rates with holograms for $H G_{n m}$ modes to that for untransformed gaussian mode: $V=\left(R_{00}-R_{m n}\right) /\left(R_{00}+R_{m n}\right)$, then for almost all of the modes with $0 \leq m, n \leq 4$ it exceeds $97 \%$, corresponding to reasonably high quality of mode transformations. Histogram of counting rates for various modes is shown in Fig. 2. Notice that counts rate for supposedly symmetrical modes $H G_{01}$ and $H G_{10}$ are visibly different. It is a consequence of different quality of projections for these modes. Such non-symmetry should be accounted for by correspondent POVM elements (namely, by the parameters $\left.\mu_{i j}^{(n m)}\right)$. This hardly controllable non-symmetry and other artifacts of non-perfect mode transformations in-

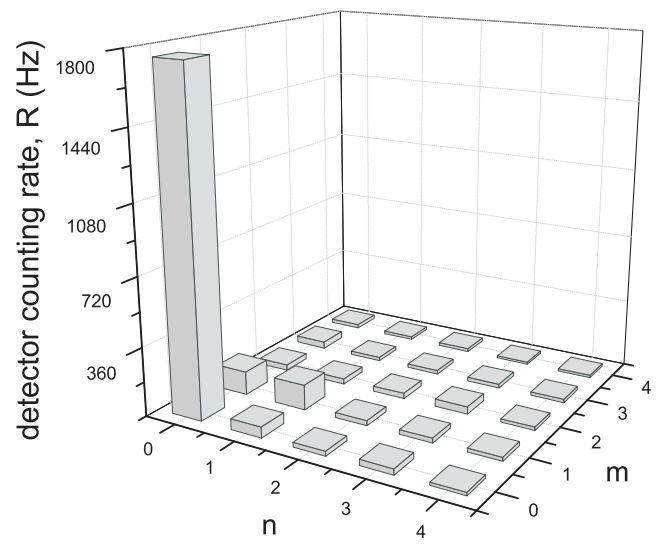

Figure 2: Detector counting rate for an attenuated laser beam transformed to $H G_{n m}$ modes. The fiber tip is set to position, corresponding to $x=0$ in Fig. 3 .

evitable with phase-only holograms is the main reason for applying the self-calibrating reconstruction procedure for the case.

To check whether the spatial structure of transformed modes is at least close to Hermite-Gaussian, we scanned the fiber tip in the focal plane of O3 objective. The counting rate dependence on fiber position is determined by the convolution of a corresponding Hermite-Gaussian function and a fundamental gaussian mode of the fiber:

$$
\begin{aligned}
& R(x) \propto \\
& \left|\int_{-\infty}^{\infty} \mathrm{H}_{n m}(\sqrt{2} \tilde{x} / w) \exp \left(-\frac{\tilde{x}^{2}}{w^{2}}\right) \exp \left(-\frac{(x-\tilde{x})^{2}}{w^{2}}\right) d \tilde{x}\right|^{2},
\end{aligned}
$$

where $w$ is the gaussian mode waist. Experimental dependencies are shown in Fig. 3 and have a characteristic shape of double-peak curves. Distance between maxima depends on the mode number, and is shown in Fig. 4 for "horizontal" $H G_{n 0}$ and "vertical" $H G_{0 m}$ modes, together with theoretical predictions for HermiteGaussian modes. To plot the theoretical predictions correctly we estimated the waist size by fitting the convolution for $H G_{00}$ mode with a gaussian curve, obtaining $w=(3.87 \pm 0.07) \mu \mathrm{m}$.

When the attenuated laser beam is substituted with SPDC radiation, the described scheme realizes projective measurements in approximately Hermite-Gaussian basis. Full scheme of experimental setup is shown in Fig. 5. Pump was focused to a $2 \mathrm{~mm} \mathrm{BBO}$ crystal with a $150 \mathrm{~mm}$ quartz lens L1, a second lens L2 with $F=145 \mathrm{~mm}$ was set confocal with L1 to collimate the beam. Pump radiation was cut off with a UV-mirror UVM, and SPDC radiation was frequency filtered with an interference filter IF. We used filters with central wavelength of $650 \mathrm{~nm}$ and bandwidth of $40 \mathrm{~nm}$ and $10 \mathrm{~nm}$ and did not observe any significant improvement of visibility for narrower filter. All the following results were obtained with a wide $40 \mathrm{~nm}$ filter. Photon pairs were split with a 50/50 non-polarizing 
(1)

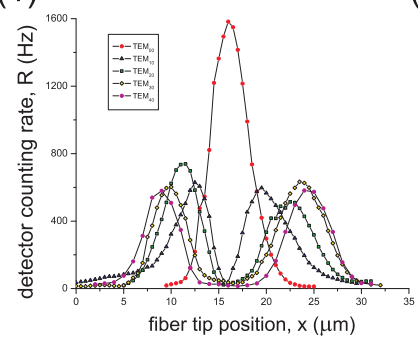

(2)

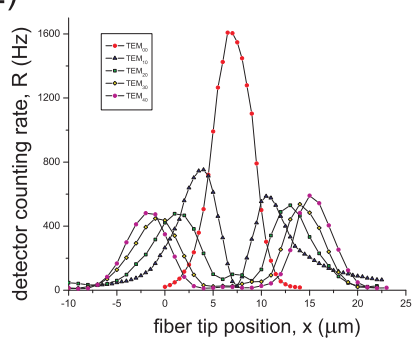

Figure 3: Counting rate dependence on the position of fiber tip for an attenuated laser beam transformed to various modes. Fiber is scanned in horizontal direction (1) and in vertical one (2).

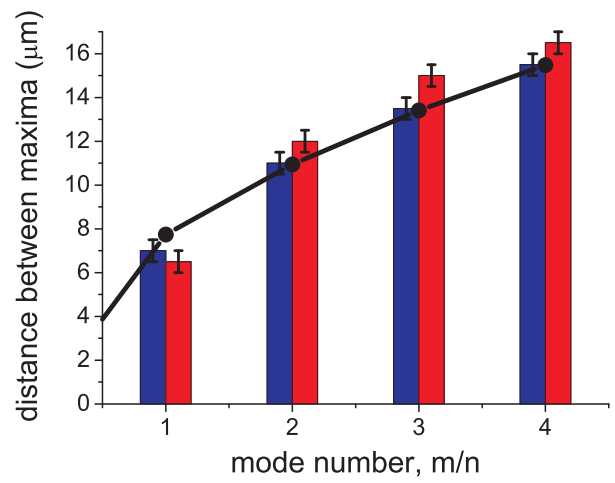

Figure 4: Dependence of maxima positions for fiber tip scan on mode number for laser beam transformations. "Horizontal" $H G_{n 0}$ (blue bars), "vertical" $H G_{0 m}$ (red bars) modes and theoretical predictions (solid black line and dots). Theoretical predictions are calculated for gaussian waist $a=3.9 \mu \mathrm{m}$ (see text for details).

beam-splitter. An SLM was placed in the transmitted channel and after reflection the radiation was focused into single mode fiber placed in the focal plane of $8 \times$ microscope objective. In the reflected channel the beam was focused into similar single mode fiber with identical objective. Signals in both channels were detected by single photon counters connected to a coincidence circuit.

Experimental evidence of similarity of Schmidt modes to Hermite-Gaussian ones may be obtained by analyzing the dependencies of single counts and coincidences on the fiber tip position in the focal plane of the focusing microscope objective. We expect the dependence for coincidences to be described by (23). Experimental curves for the case when fiber in the transmitted channel is scanned are shown in Fig. 6. Distance between maxima behaves analogously to the case of attenuated laser beam, as shown in Fig. 8 ,

We obtained same dependencies of coincidence counting rate when the fiber tip was scanned in the reflected channel (see Fig. 7). In this case single counts, obviously, do not depend on the mode selected in the conjugate channel at all. This effect is a straightforward consequence of intermodal correlations in SPDC and may

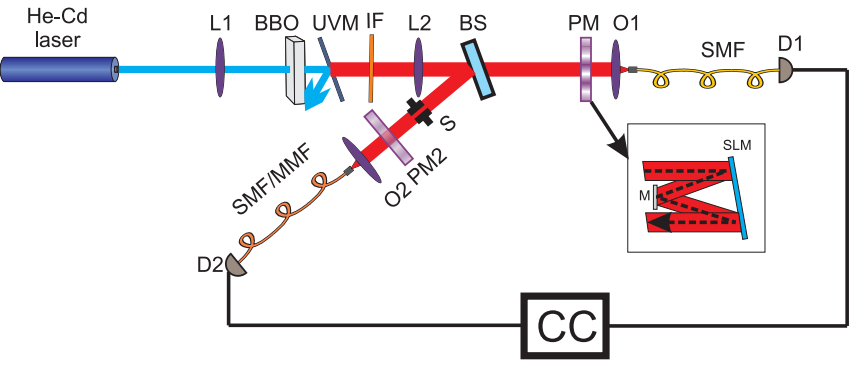

Figure 5: Experimental setup. L1 - $150 \mathrm{~mm}$ quartz lens; L2 - $145 \mathrm{~mm}$ lens; $\mathrm{BBO}-2 \mathrm{~mm}$ BBO crystal placed in the joint focus of L1 and L2; UVM - UV mirror cutting off the pump; IF - interference filter for $650 \mathrm{~nm}$ with $40 \mathrm{~nm}$ bandwidth; BS - non-polarizing 50/50 beam-splitter; 01,2 - 8× microscope objectives; PM - spatial light modulator (is shown as transmitting mask for simplicity, real alignment is shown on the inset); PM2 - phase mask made of thin glass plates; SMF single mode fiber; SMF/MMF - single or multi-mode fiber depending on the experiment (see text for details); D1,2 single photon counters (Perkin Elmer). A $200 \mu m$ vertical slit $\mathrm{S}$ was used in "ghost" imaging experiments.
(1)

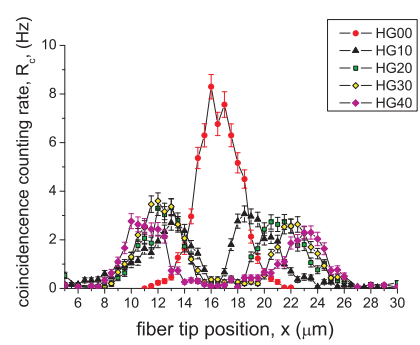

(2)

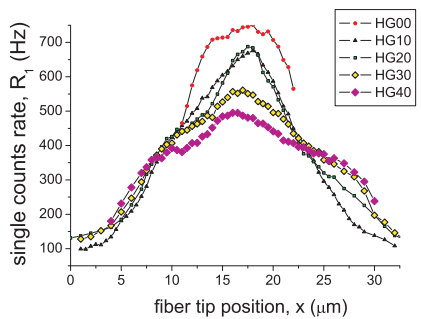

Figure 6: Coincidence (1) and single counts (2) rate dependence on the fiber tip position in the focal plane of the microscope objective in the channel with SLM for different modes. Fiber is scanned in horizontal direction.

be thought of as a sort of "ghost interference" [37]. We should note that almost zero coincidence counting rate in the central position of the fiber is an interference effect demonstrating spatial coherence of detected modes. So this result may be considered as an experimental demonstration of one of the main features of Schmidt modes their spatial coherence.

Note that the double-peak structure characteristic for Hermite-Gaussian modes appears only in coincidences dependence, while single counts behave monotonously, as is expected for spatially multi-mode radiation. The maximal value of single counting rate, however, decreases with increasing value of mode indexes. This is clear from the form of single-photon density matrix, described by (201). With the fiber placed in central position, the detection scheme in the transmitted arm of the setup realizes projections described by (21), single counts rates in this case correspond to frequencies $f_{i j}$ in (22) and are the data used for statistical inference of Schmidt eigenvalues. 


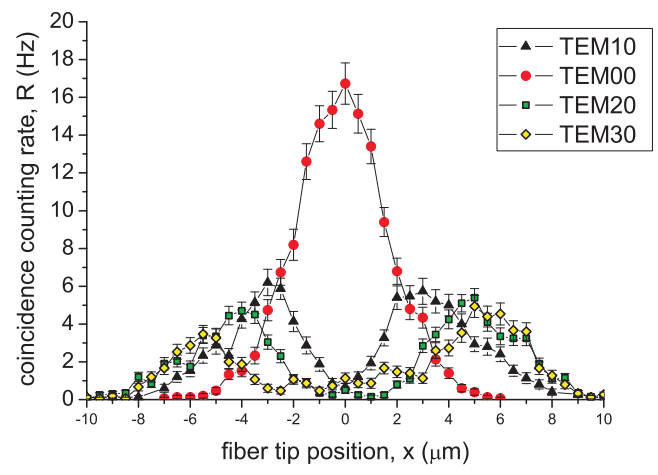

Figure 7: Coincidence counting rate dependence on the fiber tip position in the reflected channel (without SLM) for different modes. Fiber tip is scanned in horizontal direction.

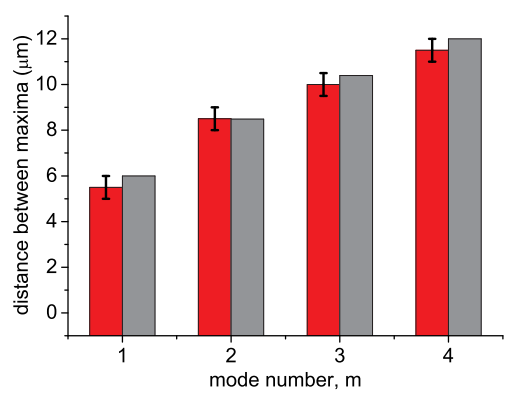

Figure 8: Dependence of maxima positions for coincidence distributions of Fig. [6 on the mode number (red bars). Calculated dependence for Hermite-Gaussian modes with $w=$ (3.0 \pm 0.1$) \mu m$ corresponding to $H G_{00}$ waist size (grey bars) is provided for comparison.

\section{PERFORMING SELF-CALIBRATION FOR INFERENCE OF SCHMIDT EIGENVALUES}

Essential features of the measurement procedure described in the previous Section can be captured by writing down parameters of POVM (21) in the following form

$$
\mu_{i j}^{(n m)} \approx \mu_{t o t} \eta_{n m} \Gamma_{i j}^{(n m)} .
$$

In Eq. (24) the parameter $\mu_{t o t}$ represent total losses (detection efficiency) equal for all measured modes. Since it contributes to normalization of the estimated signal density matrix only, it is irrelevant. The matrix $\eta_{n m}$ represents asymmetry of losses. Thus, we assume $\eta_{n m} \equiv 1$ for $n \leq m$. The parameters $\eta_{n m}$ for $n \geq m$ are to be determined via the self-calibration procedure. Parameters $\Gamma_{i j}^{(n m)}$ describe quality of the projection Since it was demonstrated that the projection is of a reasonably good quality, we assume

$$
\Gamma_{i j}^{(n m)} \approx \Delta_{m n} \delta_{i n} \delta_{j m}+d_{i j}^{(n m)},
$$

where all the parameters in the right-hand side of formula (25) are taken to be non-negative, and for each $m, n$ the

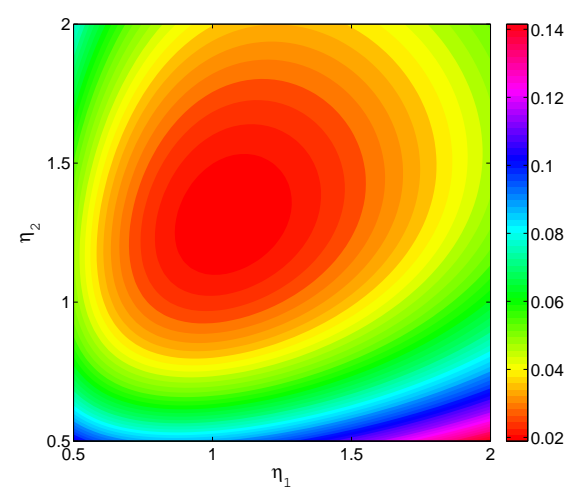

Figure 9: The Kullback-Leibler divergence (27) for the experimentally obtained frequencies.

quantity

$$
\Delta_{m n} \gg \sum_{i, j} d_{i j}^{(n m)}
$$

Also, for simplicity sake we assume that inefficiency of the projection occurs solely from contribution of other modes, so $\sum_{i, j} \Gamma_{i j}^{(n m)}=1$. Parameters $\Delta_{m n}$ can be chosen from results of projection quality measurements described in the previous Section. Thus, we have taken $\Delta_{m n}=0.97$ for all $m, n$ apart from $\Delta_{01}=\Delta_{11}=0.9$. For simulations small parameters $d_{i j}^{(n m)}$ were sampled randomly from the homogeneous distribution.

To model asymmetry of losses, we have introduced two parameters, $\eta_{1}$ and $\eta_{2}$. We assume that elements of the matrix $\eta_{n m}$ closest to the main diagonal are equal to $\eta_{1}$. Other elements of $\eta_{n m}$ for $n \geq m$ are taken to be equal to $\eta_{2}$. In Fig9 the Kullback-Leibler divergence (19) is shown for the different values of parameters $\eta_{1,2}$; for calculations Eq.(19) is recast in the standard form

$$
D(\vec{p}, \vec{f})=\sum_{i, j} f_{i j} \ln \left\{\frac{f_{i j}}{p_{i j}(\rho, X)}\right\},
$$

where both sets of estimated probabilities $\vec{p}$ and measured frequencies $\vec{f}$ are normalized to unity. The calculation is done for experimentally obtained set of frequencies shown in Fig 10(a) using the iterating ML estimaion procedure described in the Section III and given by Eq. (22). One can see that the Kullback-Leibler divergence is obviously convex for the chosen range of parameters $\eta_{1,2}$. The minimum is reached for $\eta_{1} \approx 1.3, \eta_{2} \approx 1.125$.

The result of the signal state estimation with the inferred parameters of the detecting scheme is given in Fig 10 (c). It is symmetric, i.e. $\lambda_{n m}=\lambda_{m n}$ (and it is rather different from the experimentally found frequencies shown in Fig 10(a)). However, the reconstructed set of $\lambda_{n m}$ gives a set of probabilities rather close to the measured frequencies (Fig[10(d)). The reconstruction procedure is robust with respect to small imperfections in 

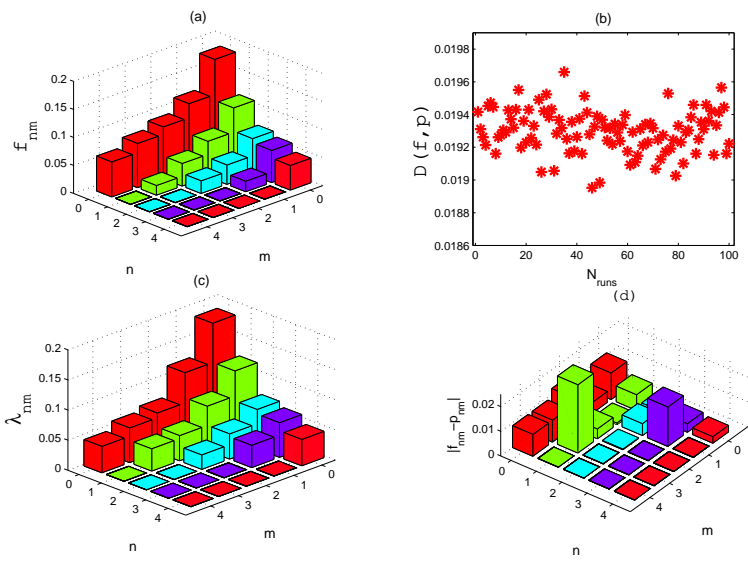

Figure 10: The panel (a) shows the registered frequencies of photocounts for different modes $H G_{n m}$. The panel (b) shows variations of the estimated minimal Kullback-Leibler divergence for different realizations of the randomly chosen parameters $d_{i j}^{(n m)} ; N_{\text {runs }}$ denoted the number of the particular realization. The panel (c) shows the the reconstructed signal density matrix elements $\lambda_{n m}$ for asymmetry parameters, $\eta_{1}=$ 1.3 and $\eta_{2}=1.125$. In the panel (d) the absolute value of differences between experimentally measured frequencies and the estimated probabilities is shown for values of parameters as for the panel (c).

performing projections. It is seen (see Fig 10(b)) that as long as the condition (26) holds, the result of the estimation changes rather weakly for different realizations of random variables $d_{i j}^{(n m)}$.

Thus, we have established that the self-calibration procedure allows one to infer with rather high precision the quantum state entering the detection scheme and parameters of this detection scheme. Now let us consider how the inferred state agrees with the double-Gaussian model developed in the Section III.

In Fig 11 one can compare estimated values of $\lambda_{0 m}$ and ones obtained using the double-Gaussian model Eq.(15) with parameters $a$ and $b$ determined in the previous Section. It can be seen that while some modeled and estimated eigenvalues are rather close, difference between other ones goes beyond estimation errors determined for MLE procedure via the Fisher information matrix [41]. The variance of the inferred element $\lambda_{n m}$ can be estimated as

$$
\Delta_{n m}=F_{n m, n m}^{-1},
$$

where the Fisher information matrix is

$$
F_{k l, m n}=\sum_{j=0}^{N} \frac{N_{m e s}}{f_{j}} \frac{\partial}{\partial \lambda_{k l}}\left[p_{j}\right] \frac{\partial}{\partial \lambda_{m n}}\left[p_{j}\right],
$$

$N_{\text {mes }}$ being the total number of measurements $(1,800$ measurements per mode was actually taken in the experiment). The estimated value of the Schmidt number, $K_{x, y}=3.34$, is reasonably close to the modelled value, $K_{x, y}^{e s t} \approx 2.97$.

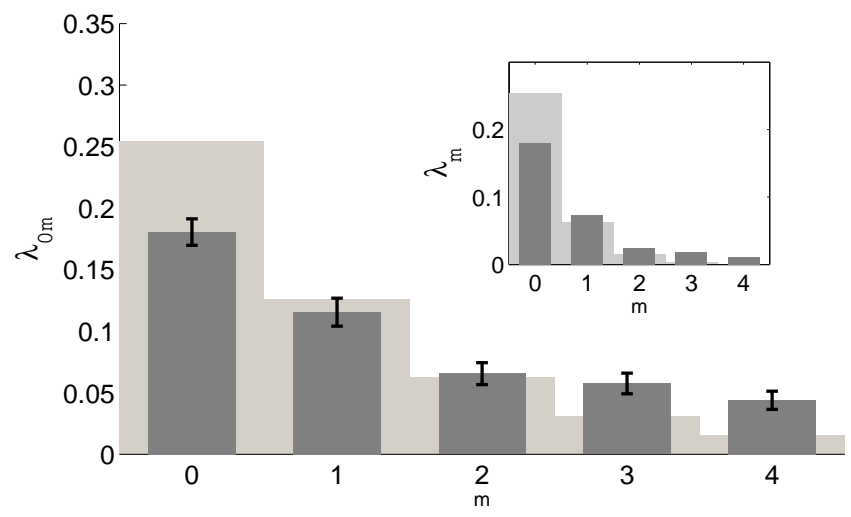

Figure 11: An estimated edge elements $\lambda_{0 m}$ (dark gray bars) and the ones modelled according to Eq. (15) (light gray bars). Errors bars represent the deviation estimated using Fisher matrix method (28). The inset shows the estimated (dark gray bars) and the modelled (light gray bars) Schmidt eigenvalues corresponding to the parameters $\frac{\lambda}{2 \pi} \times a=5.8 \times 10^{-3}$, $\frac{\lambda}{2 \pi} \times b=0.0334$.

It seems that the discrepancy can be explained, firstly, by systematic fluctuations of signal level on long time scales caused primarily by temperature fluctuations. Experiments have shown, that both coincidence and single counts rates are extremely sensitive to small beam displacements. So work of an air-conditioning system stabilizing the room temperature caused small but noticeable periodic change of signal with a period of $10 \mathrm{~min}$. Complete thermal isolation of the setup should remove this source of errors. Another significant source of errors may be the difference between detected angular aperture and full angular bandwidth of SPDC. Sharp dependence of counting rates on the position of fiber tip relatively to the microscope objective shows maximum in the position slightly different from the focal plane. Moreover, the pump, assumed to be Gaussian, was actually aberrated to $M^{2}=1.4$, which may also slightly change the real distribution.

Nevertheless, we believe our results to be providing sufficiently persuasive evidence of possibility to realize projective measurements in a basis close to spatial Schmidt modes basis for SPDC biphotons, and to perform the selfcalibrating procedure for estimating both the parameters of the measurement set-up and the signal SPDC state.

\section{CONCLUSION}

In this work we have presented the first experimentally realized self-calibrating procedure for simultaneous estimation of the signal SPDC state and imperfections of the detecting set-up. To this end we have analyzed spatial entanglement in SPDC in terms of spatial Schmidt decomposition and shown that under reasonable assumptions, applicable to the particular experiment, these modes are close to Hermite-Gaussian modes. Using this fact we have experimentally realized a scheme of projective mea- 
surements in approximately Hermite-Gaussian basis using an active spatial light modulator. Experimental results prove high quality of Gaussian beam transformation to higher order Hermite-Gaussian modes. For a spatial multi-mode SPDC radiation such spatial filtering allowed us to realize projective measurements approximating projections in Schmidt basis.

We have developed the self-calibrating protocol for the case and have demonstrated that the predicted probabilities are quite close to the registered frequencies. It clearly demonstrates validity of the self-calibration procedure performed for the case. Despite some discrepancies between the predictions of the double-Gaussian model for the Schmidt modes and the the results of the inference on the basis of the experimentally measured data, one can also claim that the double-Gaussian model is a good choice for modeling actual Schmidt modes of the generated SPDC state. Thus, we conclude that the self-calibrating procedure can become a powerful tool for updating information about the measurement itself while performing the diagnostics of the generated non-classical state.

We are grateful to M. V. Fedorov for stimulating discussions. This work was supported in part by Federal Program of the Russian Ministry of Education and Science (grant 8393), European Union Seventh Framework Programme under grant agreement n 308803 (project BRISQ2), ERA.Net RUS Project NANOQUINT, grant for the NATO project EAP.SFPP 984397 "Secure Communication Using Quantum Information Systems" and by RFBR grant 12-02-31041. S. S. Straupe and I .B. Bobrov are grateful to the "Dynasty" foundation for financial support. This work was also supported by the Foundation of Basic Research of the Republic of Belarus, by the National Academy of Sciences of Belarus through the Program "Convergence", by the Brazilian Agency FAPESP (project 2011/19696-0) (D.M.)
[1] D. N. Klyshko, Sov. J. Quantum Electron. 10, (1980) 1112.

[2] A. A. Malygin, A. N. Penin, A. V. Sergienko, Sov. Phys. JETP Lett. 33, (1981) 477 .

[3] G. M. D'Ariano, L. Maccone, and P. LoPresti, Phys. Rev. Lett. 93, (2004) 250407.

[4] G. Brida, M. Genovese, M. Gramegna, Las. Phys. Lett.3, 115 (2006).

[5] S.V. Polyakov and A.L. Migdall, Opt. Express 15, 1390 (2007).

[6] D. Mogilevtsev, J. Rehacek, and Z. Hradil, Phys. Rev. A 79, (2010) 02010(R).

[7] D. Mogilevtsev, Phys. Rev. A 82, (2010) 021807(R).

[8] D. Mogilevtsev, J. Rehacek, and Z. Hradil, New J.Phys. 14, (2012) 095001.

[9] A. M. Branczyk, D. H. Mahler, L. A. Rozema, A. Darabi, A. M. Steinberg and D. F. V. James, New J.Phys. 14, (2012) 085003.

[10] J. C. Howell, et. al., Phys.Rev.Lett. 92, (2004) 210403.

[11] M. N. OSullivan-Hale et al., Phys.Rev.Lett. 94, (2005) 220501.

[12] L. Neves et al., Phys.Rev.Lett. 94, (2005) 100501.

[13] M. V. Fedorov et al., Phys.Rev.Lett. 99, (2007) 063901.

[14] A. Mair, A. Vaziri, G. Weihs and A. Zeilinger, Nature, 412, (2001) 313.

[15] S. P. Walborn, A. N. de Oliveira, R. S. Thebaldi and C. H. Monken, Phys. Rev. A., 69, (2004) 023811.

[16] J. Leach et al., Phys. Rev. Lett., 88, (2002) 257901.

[17] A. Vaziri, G. Weihs and A. Zeilinger, Journal of Modern Optics, 89, (2002) 240401.

[18] A. Vaziri et al., Phys.Rev.Lett., 91, (2003) 227902.

[19] G. Molina-Terriza, J. P. Torres and L. Torner, Phys.Rev.Lett., 88, (2002) 013601.

[20] N. K. Langford et al., Phys. Rev. Lett., 93, (2004) 053601.

[21] S. S. R. Oemrawsingh et al., Phys. Rev. Lett., 95, (2005) 240501.

[22] B. Jack et al., Phys. Rev. A., 81, (2010) 043844.

[23] W. H. Peeters, E. J. K. Verstegen and M. P. van Exter,
Phys. Rev. A., 76, (2007) 042302.

[24] H. Di Lorenzo Pires, H. C. B. Florijn and M. P. van Exter, Phys. Rev. Lett., 104, (2010) 020505.

[25] Anand Kumar Jha et al., Phys. Rev. Lett., 104, (2010) 010501.

[26] C. K. Law, and J. H. Eberly, Phys. Rev. Lett., 92, (2004) 127903.

[27] S. S. Straupe et al., Phys. Rev. A, 83, (2011) 060302(R).

[28] D.N.Klyshko. Photons and Nonlinear Optics, (Gordon and Breach, London, 1988).

[29] A. V. Burlakov et al., Phys. Rev. A 56, (1997) 3214.

[30] C. H. Monken, P. H. S. Ribeiro, and S. Padua, Phys. Rev. A. 57, (1998) 3123.

[31] S. P. Walborn, A. N. de Oliveira, and C. H. Monken, Phys. Rev. Lett. 90, (2003) 143601.

[32] M. V. Fedorov, et al., Phys. Rev. A., 77, (2008) 032336.

[33] M. V. Fedorov, et al., Phys. Rev. A., 69, (2004) 052117.

[34] M. V. Fedorov, Yu. M. Mikhailova and P. A. Volkov, J.Phys.B:At.Mol.Opt.Phys., 42, (2009) 175503.

[35] G. F. Calvo, A. Picon and A. Bramon, Phys. Rev. A, 75, (2007) 012319.

[36] F. M. Miatto, T. Brougham and A. M. Yao, arXiv:1111.6449 [quant-ph], (2011).

[37] M. DAngelo, Y.-H. Kim, S. P. Kulik and Y. Shih, Phys. Rev. Lett., 92, (2004) 233601.

[38] Z. Hradil, Phys. Rev. A. 55, R1561 (1997); J. Fiurasek, Z. Hradil, Phys. Rev. A 63, R020101 (2001); J. Řeháček, Z. Hradil, Phys. Rev. Lett. 88, 130401 (2002) J. Řeháček, Z. Hradil, M. Zawisky, W. Treimer, M. Strobl, Europhys. Lett. 59694 (2002); Z. Hradil, J. Řeháček, Fortschr. Phys. 491083 (2001); M. Jezek, J. Fiurasek, Z. Hradil, Phys. Rev. A 68, 012305 (2003).

[39] A. P. Dempster, N.M. Laird, and D. B. Rubin, J. R. Stat. Soc. Ser. B Methodol. 39, 1 (1977); Y. Vardi and D. Lee, J. R. Stat. Soc. Ser. B Methodol. 55, 569 (1993).

[40] A.R. Rossi, S. Olivares, M.G.A. Paris, Phys. Rev. A 70, 055801 (2004); A.R. Rossi and M.G.A. Paris, Eur. Phys. J. D 32, 223 (2005); G. Zambra, A. Andreoni, M. Bondani, M. Gramegna, M. Genovese, G. Brida, A. Rossi, 
and M.G.A. Paris, Phys. Rev. Lett. 95063602 (2005).

[41] Z. Hradil, D. Mogilevtsev, and J. Rehacek, New J. Phys. 10, 043022 (2008).

[42] We have tried several criteria for choosing $\gamma$, for example equality of FWHM for both functions, which is used in [34] and gives $\gamma=0.249$. However, $\gamma=0.86$ better describes experimental data, which is not completely understood, but may be caused by limited angular detection aperture.

[43] One should pay attention to the fact, that all formulas in previous sections do not take into account the refraction on the crystal surface, so to compare with experiment all angular variables for the pump should be divided and for SPDC multiplied on $n_{e}\left(\lambda_{p}\right)=n_{o}\left(\lambda_{s}\right)=1.667$. 